TMS2014 Annual Meeting Supplemental Proceedings TMS (The Minerals, Metals \& Materials Society), 2014

\title{
CHACTERIZATION OF CU TUBE FILLED WITH AL ALLOY FOAM BY MEANS OF X-RAY COMPUTER TOMOGRAPHY
}

\author{
Girolamo Costanza ${ }^{1}$, Francesco Mantineo $^{2}$, Andrea Sili ${ }^{2}$, Maria Elisa Tata ${ }^{1}$ \\ ${ }^{1}$ Department of Industrial Engineering, University of Rome-Tor Vergata - Italy \\ ${ }^{2}$ Department of Electronic Engineering, Industrial Chemistry and Engineering, \\ University of Messina - Italy
}

Keywords: $\mathrm{Cu}$ tube, $\mathrm{Al}$ foam filler, X-Ray tomography, Compressive behaviour

Abstract

Copper tubes filled with aluminium foams were prepared by directly foaming metal powder compacts inside them. Compressive behaviour and foam-shell interface, that characterizes mechanical properties of reinforced tubes, were investigated by means of variable focus X-ray computer tomography. Compression tests were performed on empty and filled samples at increasing deformation steps: at each stage the samples were observed by tomography. A geometric evaluation of porosity on $2 \mathrm{D}$ sections was performed by calculating, for each pore, its area, equivalent diameter and circularity.

\section{Introduction}

Starting from the last years, many vehicles incorporate deformable energy absorbing elements in order to increase passive safety during impacts. These elements give rise to crushable zones and have to manage the collision energy for the rigid passenger cell protection, reducing also the deceleration. Thin-walled metallic tubes are utilized for this purpose because of their lightness and progressive buckling during deformation under axial compressive load $[1,2]$.

Cellular structures, such as honeycombs and foams, are also considered as suitable materials for energy absorption. Several of the engineering properties of metal foams are superior to those of polymeric foams; they are stiffer by an order of magnitude and stable at elevated temperatures, they possess superior fire resistance and do not evolve toxic fumes in case of fire.

Because of low density and melting temperature the main attention is paid to foams made of aluminum and its alloys. These materials show a distinct plateau of almost constant stress in the uniaxial compressive stress-strain curve, with nominal strain value up to $80 \%$, which indicates a high energy absorption capacity $[3,4]$. Their compressive properties, such as elastic modulus and plastic plateau stress, depends on cells structures $[5,6]$, in particular the amount of the absorbed energy is directly related to the way in which the cells structure is manufactured [7] and collapses $[8,9]$ under applied load. Deformation rate is another important parameter which must be monitored because compressive results may be much different in static rather than dynamic compressive tests [10].

On this basis, light metal foams has been considered as a useful filling reinforcements to improve deformation and impact behaviour of protection systems and reduce disturbing noise and vibration. The crushing load of filled tubes increases respect to the ones of empty tube and foam considered individually, due to the encroachment of the tube wall into the foam filler which modifies buckling mode and retards sectional collapse [11].

Recently many efforts have been addressed to enhance the above said properties developing foam-filled tubular structures [12], so aluminium foams have been produced and experimented 
as concern as their effects on impact energy absorption in reinforced components [13]. With the aim of understanding the mechanism of energy absorption improvement, numerical simulation of crushing due to progressive buckling under compression was carried out in [14].

However the cells collapse and the interaction between filler and tube wall surface play an important role during deformation, dependently on foaming process: casting methods, in general, are not able to create an interface bond, because the continuous aluminium oxide skin covering the foam prevents any surface reaction with the molten metal; on the contrary, the in situ foaming should facilitate the interface reaction with the formation of real metallurgical bonding. In particular it was observed [15] that direct foaming could produce an extensive bonding between aluminum foam and tube wall, if the filling process is opportunely carried out to hinder the oxide layer formation.

An investigation of cells evolution during deformation requires laborious preparations of crosssectional samples at increasing compression steps. Moreover sample needs to be progressively cut in order to take two dimensional images at different thickness, by which a three dimensional reconstruction can be performed [16]. A procedure so heavy can be avoided performing a tomographic analysis.

In a previous paper [17] an $\mathrm{Al}$ foam with closed cell, produced by powder method, was examined at various compression steps by variable focus X-ray computer tomography, in order to observe the evolution during deformation of cells morphology and their final collapse. In this work, the tomographic method was carried out to study the compressive behaviour of a copper tube, filled with aluminium by foaming directly metal powder compacts inside it.

\section{Materials and Methods}

\section{Tube and Filler Preparation}

Al foam samples have been manufactured and characterized in our laboratory. Precursor production by the powder-method has been reported in details in a previous paper [18]. In our study it has been employed $\mathrm{TiH}_{2}$ (average diameter $\left.5 \mu \mathrm{m}\right)$ as blowing agent and $\mathrm{SiC}(37 \mu \mathrm{m})$ as stabilizing agent for $\mathrm{Al}$ powder $(45 \mu \mathrm{m})$. The composition is: $\mathrm{TiH}_{2}(0.4 \mathrm{wt} \%), \mathrm{SiC}(2.8 \mathrm{wt} \%)$ and Al (to balance). After mixing for 10 minutes with a specific device, the mixture has been successively compacted by a hydraulic press (load 12 ton) into a mould to obtain a precursor (diameter $15 \mathrm{~mm}$, height $8 \mathrm{~mm}$ ) ready to be foamed into the furnace. Successively the precursor was introduced into a copper tube and the whole system heated at $700{ }^{\circ} \mathrm{C}$ in the furnace under protective Ar atmosphere. About 300 seconds is the time required for foaming in a copper tube. This combination of temperature and time permanence in the furnace has been chosen to obtain a good compromise between melt viscosity and drainage. The copper hollow tube has been treated at the same temperature $\left(700{ }^{\circ} \mathrm{C}\right)$ for the same time $(300 \mathrm{~s})$. This thermal treatment has been chosen in order to allow a direct comparison of the mechanical properties between the hollow tube and the tube filled with $\mathrm{Al}$ foam.

\section{$\underline{\text { Compression Test }}$}

Compression tests were carried out on a cylindrical sample $(\phi=16 \mathrm{~mm}, \mathrm{~h}=40 \mathrm{~mm})$ both of the hollow tube and the tube filled with the $\mathrm{Al}$ foam. Results of tests on $\mathrm{Al}$ foam samples, reported in [17], are also recalled.

The parallelism between the two platens was adjusted through the examination of the contact surface between them before the compression test. 
For each sample, the compression test was performed with steps of increasing deformation: at each step the sample was loaded, unloaded and observed by computer tomography.

\section{Tomographic Observations}

The cells morphology evolution during compression was investigated by means of a variable focus X-ray computer tomograph (CT). The tomographic system utilized works in the range microfocus-macrofocus, changing the spot size from 250 to $800 \mu \mathrm{m}$, in order to give priority to spatial resolution (between 30 and $200 \mathrm{um}$ ), penetration capacity in materials with high absorption (up to $10 \mathrm{~mm}$ in the case of ferrous alloys) or object under examination size (diameter $180 \mathrm{~mm}$, height $250 \mathrm{~mm}$ ). The X-rays are generated by a Cu tube with acceleration voltage up to a maximum of $225 \mathrm{kV}$; the emitted beam spectrum is limited, by means of metallic filters (Al, $\mathrm{Cu}, \mathrm{Sn}, \mathrm{Pb}$ ), in a narrow frequency range to avoid problems related to background noise; the $\mathrm{X}$ rays detector (flat panel) consists of a two-dimensional array of crystals and CCD sensors.

The operating parameters choice is carried out by optimizing scan time, spatial resolution and contrast. The object to be inspected is rotated inside the radiation cone beam produced by the Xray source. The intensity distribution of the radiation transmitted through the work-piece is measured by means of a flat panel detector and digitally stored. After recording all the projection data during a full rotation, the volumetric model of sample, with high point densities (several million points), is obtained by a computerized 3D reconstruction.

\section{Image Analysis}

From the reconstructed volume, 2D sections of the observed samples were considered. These images were processed to enhance contrast and brightness and then transformed into their negative. On such images a threshold value was defined for classifying each pixel as belonging to wall or empty zone of the cell. A geometric evaluation of porosity was performed by calculating for each cell its area, equivalent diameter, perimeter and circularity. The cell area A is calculated from the number of pixel counted in a single cell.

The equivalent diameter is given by the following relationship:

$D_{\text {eq }}=\sqrt{ } 4 \mathrm{~A} / \pi$

The average perimeter is calculated by the Crofton relationship:

$\mathrm{P}=\pi\left[\mathrm{P}_{0^{\circ}}+\mathrm{P}_{45^{\circ}}+\mathrm{P}_{90^{\circ}}+\mathrm{P}_{135^{\circ}}\right] / 4$

where $\mathrm{P}_{0^{\circ}}+\mathrm{P}_{45^{\circ}}+\mathrm{P}_{90^{\circ}}+\mathrm{P}_{135^{\circ}}$ are perimeters measured on four projections of the cell.

The circularity is given by the following relationship:

$\mathrm{C}=4 \pi \mathrm{A} / \mathrm{P}^{2}$

The geometric evaluation of porosity during compression test was performed on representative sections of the samples, recorded at each compression step. 


\section{Results and Discussion}

The loading-unloading curves of each compression step were assembled obtaining compression diagrams for both the annealed hollow tube and the tube filled with Al foam. These diagrams are given in fig. 1 together with the compressive behaviour of a cylindrical sample of the $\mathrm{Al}$ foam. The compressive behaviour of the Al foam sample (diagram a) is characterized by a very short initial region of linear elasticity, followed by a roughly constant plateau at low stress levels, related to plastic deformation, and finally by a stage of sharp stress increasing, ascribed to a widespread cells collapse [17].

The annealed hollow tube behaviour (diagram b) is characterized by buckling phenomena with peaks of load corresponding at each fold formation.

The compression response of the tube filled with the Al foam (diagram c) shows a significant improvement of the absorbed energy, that can be ascribed to interaction phenomena between $\mathrm{Al}$ foamed core and $\mathrm{Cu}$ shell. In particular a greater number of peaks of load can be observed.

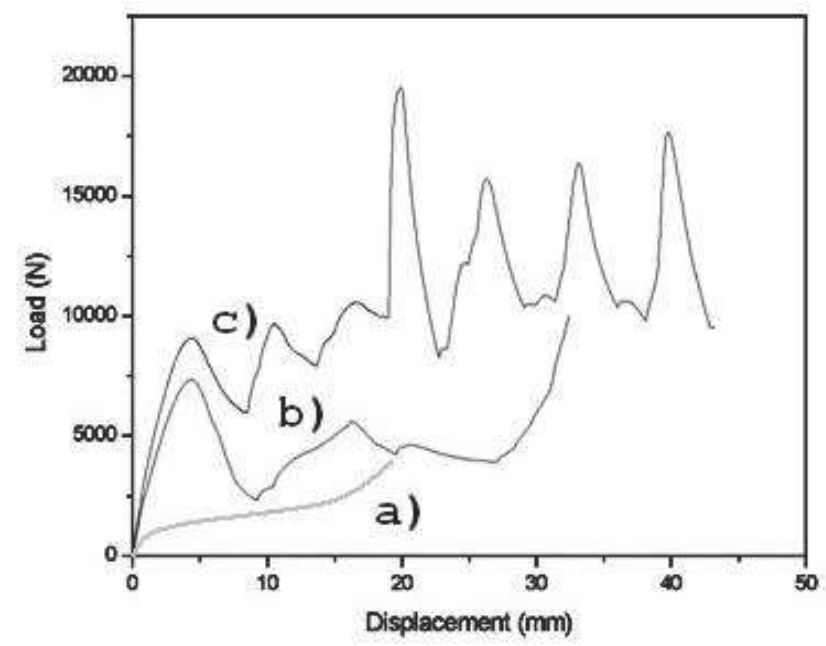

Fig. 1 - Compression diagrams: a) Al foam sample, b) $\mathrm{Cu}$ hollow tube, c) $\mathrm{Cu}$ tube filled with $\mathrm{Al}$ foam.

The annealed hollow tube show an axis-symmetric collapse mode (curve b), named "concertina", typical of plastic materials, where deformation features as well as number and pattern of folds depend on geometry and material properties [19].

Compared to the empty tube the buckling pattern of the foam filled specimen is noticeably different being the folds forced more outwards and placed at shorter distances (see the distance between peaks of the diagrams b) and c) in fig. 1).

Moreover, as shown in fig. 2 a), the empty tube started to buckle at one of the two ends and continued sequentially. On the contrary, in the filled tube buckling started with folds at both the two ends of the sample while the third fold, as can be observed in fig. 2 b), grew at half height. 


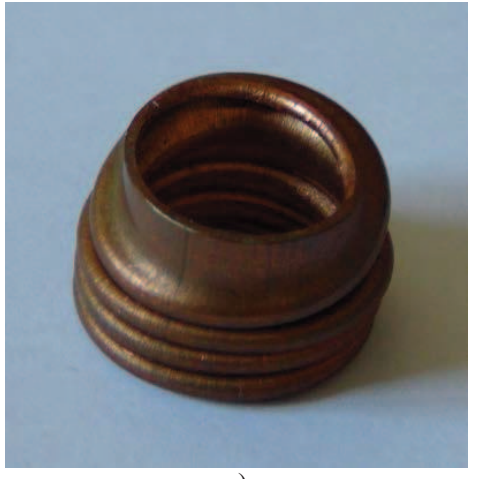

a)

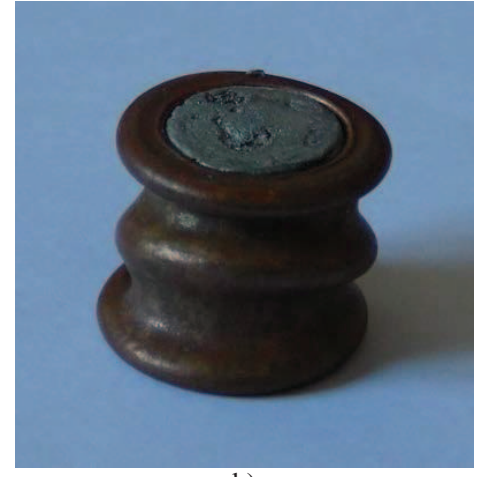

b)

Fig. 2 - Deformed samples: a) annealed hollow tube after full deformation; b) tube filled with $\mathrm{Al}$ foam after the third fold formation.

The CT images in fig. 3 show a 2D section of the filled tube not deformed and after two different levels of deformation. Due to the interaction between foam and tube wall, the reinforcement intrudes, even if partially, into the tube folds, reaching conditions of multiaxial state of compression. However the foam does not penetrate completely into the tube folds because of bonding not sufficiently able to transfer tangential stresses to the core, reducing the "interaction effect" and consequently the absorbed energy.

So an important role for energy absorption has to be ascribed to the interaction between interfaces. Its improvement, as pointed out in [15], can be reached performing the production process in a fully non-oxidizing atmosphere.

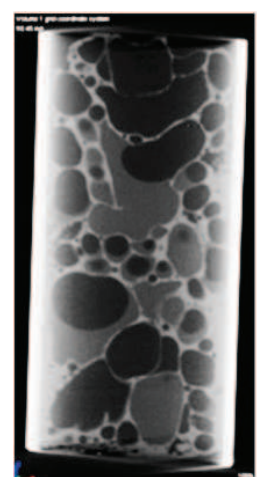

a)

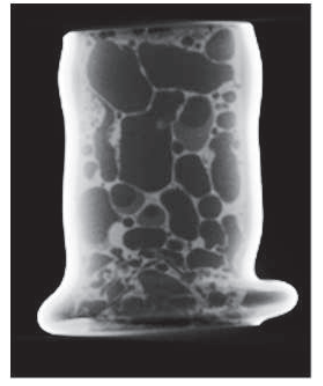

b)

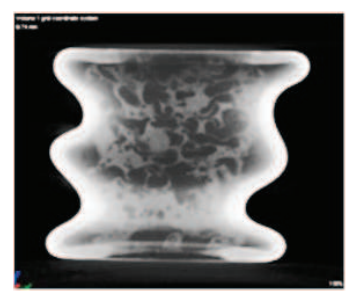

c)

Fig. 3 - Al foam filled tube: tomographic images of the same section before compression (a) and after folds formation $(b, c)$. 
Results of the geometric evaluation of cells during compression are expressed through the equivalent diameter and circularity, calculated by means of equation (1) and (3) respectively. These calculations are performed on different sections for each sample in order to have results statistically more significant.

Figure 4 and 5 shows respectively the histograms of the cells number according to the equivalent diameter values and according to the circularity values, for a not deformed sample and after two levels of compression.

Going on compression, cells reduce progressively their dimensions: at each compression step, the number of cells with a small equivalent diameter increase, while all the cells with a great equivalent diameter disappear.

In addition to the equivalent diameter reduction, compression causes changes of the cells morphology, because most of the cells lose their spherical shape: in the not deformed samples there is a great number of cells with circularity near to 1 , while with the increasing of deformation the number of spherical cells is reduced significantly.

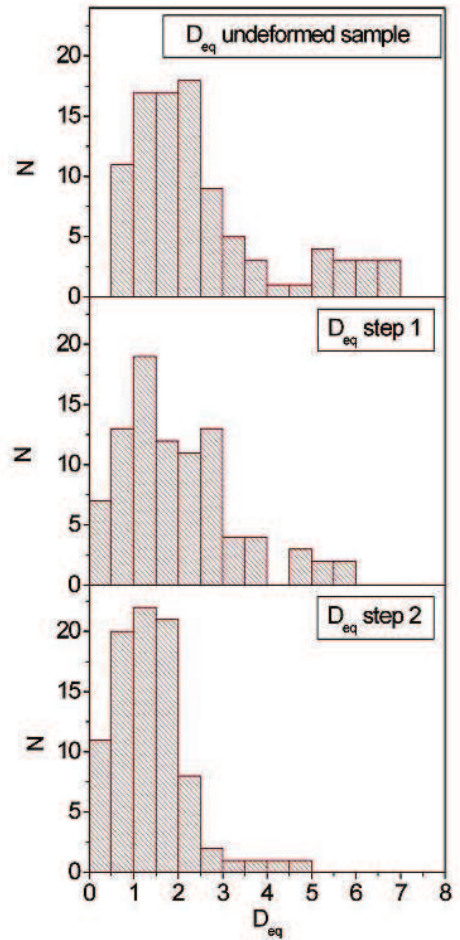

Fig. 4 - Histograms of the cells number N vs. the equivalent diameter values $\mathrm{D}_{\text {eq }}(\mathrm{mm})$.

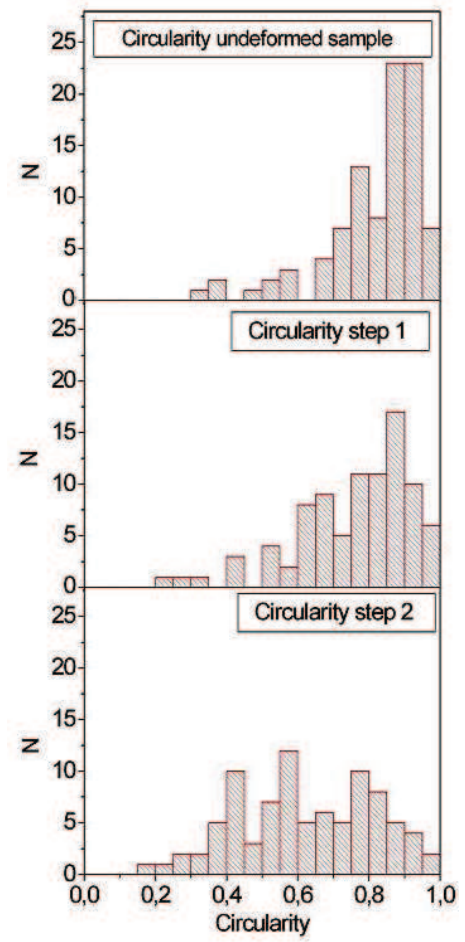

Fig. 5 - Histograms of the cells number $\mathrm{N}$ vs. the values of circularity $\mathrm{C}$. 


\section{Conclusions}

Compression tests were performed both on copper hollow tube and tube filled with $\mathrm{Al}$ foam at increasing deformation steps. Compared to the empty tube, the buckling pattern of the foam filled specimen is noticeably different being characterized by a greater number of folds that are forced more outwards and placed at shorter distances. First of all the X-ray computerized tomography analysis showed that the reinforcement intrudes, even if partially, into the tube folds absorbing the deformation energy. Moreover, at each compression step it can be observed the disappearance of cells with the greatest equivalent diameter and changes of the cells morphology, which lose their spherical shape. These phenomena determine that the $\mathrm{Al}$ foam filler modifies the buckling mode of the tube, retarding its sectional collapse and absorbing greater amounts of energy than in the empty sample.

\section{References}

1. G. Lu and T. Yu, "Energy absorption of structures and materials", Woodhead Publishing Limited, Cambridge, UK, (2003), 1-424.

2. G. Costanza, M.E. Tata, "Metal foams: Recent experimental results and further developments", Metallurgia Italiana, Vol. 103 n 3 (2011), 3-7.

3. L. J. Gibson, M. F. Ashby, "Cellular Solids: Structure and Properties", $2^{\text {nd }}$ edn., Cambridge University Press, Cambridge, (1997), 1-510.

4. G. Costanza, G. Gusmano, R. Montanari, M.E. Tata, "Manufacturing routes and applications of metal foams", Metallurgia Italiana, Vol. $95 \mathrm{n}^{\circ} 2$ (2003), 31-35.

5. H. Yu, Z. Guo, Bing Li, G. Yao H. Luo, Y. Liu, "Research into the effect of cell diameter of aluminium foam on its compressive and energy absorption properties", Material Science and Engineering A, 454-455 (2007), 542-546.

6. F. Campana, D. Pillone, "Effect of wall microstructure and morphometric parameters on the crush behaviour of Al alloy foams", Materials Science and Engineering A, 479 (2008), 5864.

7. G. Costanza, R. Montanari, M.E. Tata, "Optimisation of $\mathrm{TiH}_{2}$, and $\mathrm{SiC}$ content in $\mathrm{AL}$ foams", Metallurgia Italiana, Vol. $97 \mathrm{n}^{\circ} 6$ (2005), 41-47.

8. H.W. Song, Q.J. He, J.J. Xie, A. Tobota, "Fracture mechanisms and size effects of brittle metallic foams: in situ compression test inside SEM", Composites Science and Technology, 68 (2008), 2441-2450.

9. Y. Mu, G. Yao, L. Liang, H. Luo, G. Zu, "Deformation mechanims of closed-cell aluminum foam in compression", Scripta Materialia, 63 (2010), 629-632.

10. G. Costanza, M.E. Tata, "Dynamic and static compressive behaviour of aluminium foam", Proceedings of the 4th International Structural Engineering and Construction Conference, ISEC-4 - Innovations in Structural Engineering and Construction, Vol. 2 (2008) 919-922.

11. M.Seitzberger, F.G. Rammerstorf. H.P. Degischer, R.Gradinger, "Crushing of axially compressed steel tubes filled with aluminium foam”, Acta Mechanica, 125 (1997), 93-105.

12. Makoto Kobashi, Ryosuke Sato, Naoyuki Kanetake, "Foaming and filling-in behavior of porous aluminum in hollow components", Materials Transactions, V. 47, No 9 (2006), 21782182. 